\title{
ACOSO ESCOLAR Y AUTOESTIMA EN ALUMNOS DE SEGUNDO DE SECUNDARIA DE UNA INSTITUCIÓN EDUCATIVA DEL DISTRITO DE LURIN
}

\section{Bullying and self-esteem in students of the second year of secondary school from the district of Lurin}

\author{
Mayra Fernanda Cuya Zavaleta* \\ Asociación Educativa Carpe Diem Lurín - Lima, Perú \\ https://orcid.org/0000-0003-1209-2224 \\ Herbert Robles Mori** \\ Universidad Femenina del Sagrado Corazón - Lima, Perú \\ https://orcid.org/0000-0002-5937-5937
}

\begin{abstract}
Resumen
El propósito del estudio fue determinar la relación entre las dimensiones de acoso escolar y autoestima. El diseño de la investigación fue descriptivo correlacional. La población del estudio estuvo conformada por 139 alumnos de segundo de secundaria de una institución educativa del distrito de Lurín. El muestreo fue no probabilístico de tipo intencional con un total de 98 participantes. Los instrumentos utilizados fueron acoso y violencia escolar de Oñate y Piñuel, validado por Aranda en el 2010 (Alfa de Cronbach .93) e Inventario de Autoestima de Coopersmith, validado por Mesías en el 2017 (Alfa de Cronbach .799), ambos en el Perú. Los resultados muestran una correlación entre el índice global de acoso y la autoestima general con un coeficiente de correlación Spearman (-,485**). Se concluye que existe una relación significativa entre ambas variables del estudio.
\end{abstract}

Palabras clave: acoso escolar, autoestima, escalas de acoso, factores.

\begin{abstract}
The purpose of this study was to determine the relationship between the dimensions of bullying and self-esteem. The research design was descriptive correlational. The study population consisted of 139 second year secondary school students from a school in the district of Lurín. A non-probabilistic, intentional sample consisting of 98 participants was used. The instruments used were Bullying and School Violence test by Oñate and Piñuel, validated by Aranda in 2010 (Cronbach's Alpha $=0.93)$ and Coopersmith's Self-Esteem Inventory, validated by Mesías in 2017 (Cronbach's Alpha = 0.799), both in Peru. The results show a correlation between the global bullying index and general self-esteem with a Spearman correlation coefficient (-0.485**). It is concluded that there is a significant relationship between both variables.
\end{abstract}

Keywords: school bullying, self-esteem, bullying scales, factors.

Cualquier uso que se haga de este artículo debe incluir: Autor / Título original de la publicación / ISSN.

\footnotetext{
* Psicóloga.mayra_cuyaz@hotmail.com

**Psicólogo.hrobles@unife.edu.pe
} 


\section{INTRODUCCIÓN}

Aprender a convivir es sin duda alguna un reto necesario y fundamental para el desarrollo de todo ser humano, la convivencia no resulta nada fácil para las personas, y en especial para los alumnos, ya que ingresan a un mundo en donde lo pares están constantemente pendientes unos a otros, y el mínimo error de convivencia, actitudes y personalidad puede generar violencia escolar. El acoso escolar es un problema de salud ya que afecta a muchos niños y adolescentes a nivel mundial. Según la Organización Mundial de la Salud aproximadamente un $42 \%$ de escolares varones y $37 \%$ de escolares mujeres refieren haber sido víctimas de riñas físicas e intimidación,(OMS, 2016), por tal motivo la violencia escolar es considerado como una modalidad sistemática del agresor hacia su víctima, lo cual es una violencia que contribuye enormemente a la carga mundial de muertes prematuras, lesiones y discapacidad, además de tener repercusiones graves, que a menudo perduran toda la vida, en el funcionamiento psicológico y social de la persona.

Una de las principales consecuencias del acoso escolar es la baja autoestima que presentan las víctimas; algunos autores, como Oñate (2007), señalan la baja autoestima como una de las consecuencias del acoso escolar ya que generan graves problemas psicológicos y sociales, son las víctimas quienes resultan más afectadas ya que tienden a generar baja autoestima, conductas depresivas, flagelación y aislamiento.

\section{ACOSO ESCOLAR}

El concepto de acoso escolar surge de muchos autores que lo definen, uno de ellos es; Oñate (2007) quien considera al acoso escolar como un proceso de persecución, tales como; estigmatización, rechazo, burla y humillación, donde la víctima es chivo expiatorio del grupo de compañeros de clase. Asimismo, manifiesta que las consecuencias de acoso escolar generan graves problemas psicológicos y sociales, pero más afectadas son las víctimas ya que tienden a generar conductas depresivas, baja autoestima, flagelación y aislamiento. Por lo general estas víctimas experimentan pensamientos o actos suicidas, debido a la fuerte carga emocional que atraviesan, no logran comunicar dichos sucesos, simplemente los manejan por sí solos, sin buscar ayuda externa, lo cual es una agonía constante y permanente, que puede desencadenar el suicidio consumado.

\section{Otros autores también lo definen como:}

Violencia sostenida, mental o física, guiada por un individuo o por un grupo y dirigida contra otro individuo que no es capaz de enfrentar, ni defenderse a sí mismo en esta situación, y conlleva a agresiones en distintas formas, como; física, verbal o indirecta, Olweus (1998).

Acoso en la escuela como una construcción social que abarca aspectos latentes, de los cuales interrumpe y afecta el clima escolar, asimismo se contrasta esta alineación al origen subjetivo cuya función se centra en situaciones donde la dinámica está alterada y fallida, Osorio (2006).

Una forma de agresión, con intencionalidad de un estudiante a otro compañero, el cual se convierte en su víctima cotidiana, esta acción suele ser persistente, puede durar semanas, meses y hasta años, Cerezo (2002).

Existen modelos teóricos que dan sustento al acoso escolar, uno de ellos es;

La teoría propuesta por Bandura (1976) considera que el comportamiento agresivo es el resultado de un aprendizaje por observación e imitación. La imitación de la conducta agresiva dependerá de si el modelo observado obtiene o no recompensas positivas de su agresividad: si obtiene un beneficio se incrementará la probabilidad de que se imite el comportamiento agresivo, pero si el modelo es castigado por su conducta disminuirá la probabilidad de imitación (Ramos, 2008; p. 85).

Por otro lado, el acoso escolar tiene características, donde según Gonzáles (2009) el eje principal es generar daño físico y psicológico mediante la reiteración conductual agresiva durante un tiempo prolongado. Las victimas por lo general experimentan sensaciones de vacío, situación de inferioridad que tiene como consecuencia sentimientos de inseguridad y deterioro de su integridad personal, asimismo el desequilibrio 
y abuso de poder impide que este círculo vicioso termine.

Porhola y Kinney (2010) plantean que la violencia escolar genera amenaza al bienestar físico y psicosocial de los alumnos, asimismo lo definen como un comportamiento agresivo caracterizado por el desequilibrio de poder en las relaciones interpersonales donde el proceso es reiterado en base a ofensas verbales, físicas y de exclusión social. Aquí las victimas pueden sufrir dos tipos de acoso, tales como; acoso encubierto, definido así debido a que las víctimas no logran identificar a su agresor, mientras que en el acoso manifiesto si logran identificar directamente a su agresor. Estos acosos tienen acciones dañinas, tales como; golpes, empujones, patadas, insultos, ofensas, amenazas, y gestos amenazadores, su única finalidad es generar daño al otro, siendo un hecho placentero para quien lo realiza.

En el acoso escolar existen agentes, donde según Mendoza (2012) determina una triada en el acoso escolar, considerándolo como un proceso de interacción entre tres agentes, tales son; agresor, víctima y testigo. A todo ello define al agresor como un agente que intimida y acosa debido a la necesidad de poder o control, también señala a la víctima como un agente objeto de burla, ya que carecen de seguridad y falto de estrategias de enfrentamiento, por último, define al testigo como un agente que fomenta o inhibe el maltrato que observa, por lo que a su vez adopta un rol de agresor pasivo, seguidor o secuaz. Este proceso de interacción produce un círculo de victimización ya que el agresor fortalece su poder o popularidad mientras que la víctima cada vez se debilita. También existe una violencia entre pares, pero también puede desencadenarse dentro del ámbito escolar un acoso entre profesor-alumno, esto lo determina Cabeza (2007) quien señala que en ocasiones el profesor puede jugar un rol importante para superar dichos eventos de acoso escolar, pero otras situaciones participan y fomenta la agresión y el maltrato hacia sus propios alumnos. Utilizan métodos para desviar su conducta ofensiva y dañina; mayormente la justifican bajo su rol de autoridad, asimismo los alumnos victima son desvalorados, criticados y humillados delante de sus compañeros de clase, y hasta intimidados por los mismos ya que el causal es generado por el propio docente.
Existen consecuencias que afectan el desarrollo emocional, personal y social de las personas, frente a ello Cleary, Sullivan \& Sullivan (2003) determinan que el acoso escolar es un comportamiento impredecible, el cual genera problemas y daños al núcleo escolar. Asimismo, afirman que esta problemática ha surgido en los últimos veinte años, y es por ello que los expertos de la educación han centrado su interés para lograr entender este complejo comportamiento, que no distingue raza, género, ni clase, y que además produce un impacto dañino a todo el sistema educativo, pero en especial a la víctima. Del mismo modo García (2011) señala que el acoso escolar ejerce abuso de poder, donde el daño generado e infligido genera proporciones desmesuradas de daños y efectos nocivos, por lo tanto, sus consecuencias claramente demuestran perjuicio físico, psicológico o de cualquier índole donde la victima adquiere altos niveles de estrés postraumáticos

Finalmente se considera que para afrontar situaciones de acoso escolar se requiere un adecuado manejo de intervención, frente a ello Ortega y Del Rey (2003) señalan que es esencial e importante establecer este vínculo entre los alumnos y dichas normas de convivencia, ya que así se mantendría un microsistema de relaciones interpersonales entre sí. El abuso social entre compañeros rompe todo esquema o vinculo interpersonal, ya que se establece un foco dual entre agresor-víctima, que a su vez es reforzado por los espectadores más o menos activos ya que unos animan al agresor $\mathrm{u}$ otros ayudan a la víctima, del cual lamentablemente tiene consecuencias y efectos dañinos en el proceso y desarrollo de aprendizaje, valores, actitudes y normas. Hernández \& Sancho (2004) señalan que para disminuir los niveles de acoso dentro del ámbito educativo es fundamental apuntar en el clima escolar, ya que favorece en mejorar las relaciones interpersonales de los alumnos, para ello tiene que brindarse un clima acogedor entre todos sus miembros, tanto alumnos como profesores, asimismo este clima tiene como eje principal la comunicación adecuada entre todos sus miembros.

\section{AUTOESTIMA}

El concepto de Autoestima es definido por Izquierdo (2008) quien manifiesta que la autoestima es la suma de la confianza y el respeto que se debe 
sentir por uno mismo, refleja también el juicio de valor que cada uno hace de su persona para enfrentarse a los desafíos que le presenta la vida, y el derecho a ser feliz. Tener una autoestima alta es sentirse confiadamente apto para la vida, lo cual es sentirse valioso, capaz y con una adecuada aceptación personal, que le permitirá lograr un crecimiento personal óptimo.

\section{Otros autores la definen también como:}

Una estructura cognitiva de experiencia evaluativa real e ideal que el sujeto realiza de sí mismo, lo cual está condicionado socialmente en su formación y expresión, es indudablemente el valor que le brindamos a nuestro propio yo (Gonzales-Arratia, 2001).

Feldman (2005) define a la autoestima como los sentimientos y creencias que tenemos de nosotros mismos y afecta a todo lo que hacemos en la vida, es compleja, cambiante y está arraigada con nuestro ser, asimismo es la aceptación propia, conocerse, tener una idea objetiva de quiénes somos y la capacidad de enfrentarse a la vida.

Gonzales-Arratia (2001; p.48) señaló que "según Coopersmith (1967) considera que la autoestima es la evaluación que el individuo hace y mantiene por costumbre sobre sí mismo, expresa una actitud de aprobación e indica el grado en que el individuo se cree capaz, importante exitoso y valioso".

Existen modelos teóricos que dan sustento a la autoestima, uno de ellos es;

Craig (1998) Erickson presenta un modelo de ocho etapas del desarrollo, todos los hombres experimentan ocho crisis o conflictos y los ajustes que el individuo hace en cada etapa pueden ser alterados o revertidos más tarde. Considera que la autoestima se conforma en la etapa de confianza básica vs desconfianza, que resulta de vital importancia en el desarrollo de la persona (Gonzales-Arratia, 2001; p27).

Por otro lado, la autoestima tiene características, tales como lo señala Feldman (2005) las experiencias del niño y con las reacciones de los demás, si tiene experiencias de éxito y rememora una acción positiva, su autoestima aumenta, de lo contrario si sufre fracasos y reacciones negativas, entonces disminuye, es por ello que la autoestima es la combinación de experiencias, interacciones e información que proviene de afuera.

Las características de la autoestima se basan en; amor de sí verdadero y profundo, aceptación auténtica y genuina de sí misma, autoconfianza y alegría agradecida, todo ello son reflejos claros de lo que el sujeto adhiere de sí mismo (Izquierdo, 2008).

Finalmente, la autoestima en el sistema educativo según Vernieri (2006) la escuela es el inicio de oportunidad en la que los alumnos tienen que transformar la construcción de la imagen interna original a partir de lo que le devuelve otro objetivo, ese otro puede ser el maestro quien puede restituir una imagen igual, parecida o muy diferente a las que le mostraba aquel primer espejo familiar, es por ello que la escuela genera la oportunidad de reencontrarse y consolidarse a partir de la visión clara y responsable de los docentes.

El profesor dentro del sistema educativo tiene como rol ayudar a los estudiantes a identificar y valorar sus características y habilidades (el yo percibido) y también el de saber diferenciarse entre sus compañeros, siendo un sujeto único e irrepetible (Salm, 1999).

Beltrán y Bueno (1995) manifiestan que la escuela es un eje central en el desarrollo del alumno, ya que no solo contribuye con generar capacidades intelectuales sino también el de fortalecer y desarrollar la autoestima, por lo que dentro de la escuela el alumno se interrelaciona con sus compañeros y maestros quienes influyen en la valoración y reafirmación de su propio ser.

La escuela influye en la autoestima del niño, ya que genera que logre enfrentarse a mayores evaluaciones sobre la forma de pensar y actuar en contraste con la que ha tenido en casa, asimismo los alumnos tienen que hacer frente a recordatorios diarios de sus capacidades y limitaciones, recompensas, castigos, éxitos y fracasos, por lo que empezará a formarse una imagen de sí mismo a partir de lo que los otros que resulta significativo le transmiten (Gonzales- Arratia, 2001). 


\section{METODOLOGÍA}

\section{Tipo y Diseño de Estudio}

Esta investigación es de tipo básico y sustantivo, (Sánchez y Reyes, 2015) es básico porque los resultados obtenidos aportan al campo del conocimiento científico al que pertenece, que en este caso se trata del área educativa por tratarse de alumnos de segundo año del nivel secundario. Según los autores mencionados, esta investigación también es sustantiva, ya que describe y explica la realidad del acoso escolar en el ámbito educativo, con lo cual se pretende brindar algunos aportes que faciliten su abordaje desde una visión psicológica, asimismo la investigación es un diseño descriptivo correlacional.

\section{Variables del estudio}

1. Acoso escolar (índices globales, escala de acoso, factores y escalas clínicas)

2. Autoestima (general, social, escolar y hogar)

\section{Población y Muestra de estudio}

La población para la investigación fueron los alumnos de segundo de secundaria de una institución educativa del distrito de Lurín, con un total de 139; el tipo de muestreo fue no probabilístico de tipo intencional, con un total de 98 alumnos de ambos sexos, los cuales se detalla a continuación.

\section{Tabla 1}

Género de alumnos de segundo grado de secundaria

\begin{tabular}{lcc}
\hline Género & $\mathrm{f}$ & $\%$ \\
\hline Femenino & 48 & $49.0 \%$ \\
Masculino & 50 & $51.0 \%$ \\
\hline
\end{tabular}

Nota: $\mathrm{N}=98$

En la tabla 1, se puede apreciar que los alumnos de sexo masculino tienen una frecuencia de $50(51,0 \%)$ y las alumnas de género femenino una frecuencia de $48(49,0 \%)$.

\section{Instrumentos}

Para la siguiente investigación se utilizó Acoso y violencia escolar de Oñate y Piñuel (2012) validado en alumnas de primero de secundaria de una institución educativa del Callao - Perú por Aranda en el año 2006, Alfa de Cronbach de (.93), lo cual indica que el instrumento es confiable.

Para la evaluación de Autoestima se utilizó Inventario de Autoestima de Coopersmith (1967) validado en adolescentes de la provincia de HuallagaPerú por Mesías en el año 2017, Alfa de Cronbach (.799), lo cual indica que el instrumento es confiable.

\section{Procesamiento Estadístico}

Para la siguiente investigación se utilizó:

a) Estadística descriptiva: tabla de frecuencias y porcentajes.

b) Estadística inferencial: Coeficiente de correlación Spearman.

\section{RESULTADOS \\ RESUTTAOS}




\section{Tabla 2}

Coeficientes de correlación entre Violencia escolar y Autoestima

\begin{tabular}{|c|c|c|c|c|}
\hline & \multicolumn{4}{|c|}{ Autoestima } \\
\hline & General & Social & Escolar & Hogar \\
\hline \multicolumn{5}{|l|}{ Índices globales } \\
\hline Índice global de acoso &,$- 485^{* *}$ &,$- 357^{* *}$ &,$- 423 * *$ &,$- 446 * *$ \\
\hline Intensidad de acoso &,$- 389 * *$ &,$- 212 *$ &,$- 435 * *$ &,$- 376 * *$ \\
\hline \multicolumn{5}{|l|}{ Escala de acoso } \\
\hline Hostigamiento &,$- 379 * *$ &,$- 294 * *$ &,$- 358 * *$ &,$- 346 * *$ \\
\hline Intimidación &,$- 276^{* *}$ &,- 088 &,$- 279 * *$ &,$- 293 * *$ \\
\hline Amenazas &,$- 231 *$ &,$- 253 *$ &,$- 225^{*}$ &,$- 271 * *$ \\
\hline Coacciones &,$- 244^{*}$ &,- 061 &,$- 239 *$ &,$- 230 *$ \\
\hline Bloqueo social &,$- 316 * *$ &,$- 328 * *$ &,$- 324 * *$ &,$- 254 *$ \\
\hline Exclusión social &,$- 335^{* *}$ &,$- 266^{* *}$ &,$- 371 * *$ &,$- 303 * *$ \\
\hline Manipulación social &,$- 391 * *$ &,$- 286^{* *}$ &,$- 288 * *$ &,$- 392 * *$ \\
\hline Agresiones &,$- 364 * *$ &,- 192 &,$- 263 * *$ &,$- 396 * *$ \\
\hline \multicolumn{5}{|l|}{ Factores } \\
\hline Factor Hostigamiento &,$- 396^{* *}$ &,$- 288^{* *}$ &,$- 346 * *$ &,$- 339 * *$ \\
\hline Factor intimidación &,$- 307 * *$ &,$- 199 *$ &,$- 380 * *$ &,$- 397 * *$ \\
\hline Factor exclusión &,$- 510 * *$ &,$- 356^{* *}$ &,$- 373 * *$ &,$- 392 * *$ \\
\hline Factor agresiones &,$- 327 * *$ &,$- 210 *$ &,$- 292 * *$ &,$- 416 * *$ \\
\hline \multicolumn{5}{|l|}{ Escalas clínicas } \\
\hline Ansiedad &,$- 557 * *$ &,$- 262 * *$ &,$- 276 * *$ &,$- 256^{*}$ \\
\hline Estrés postraumático &,$- 581 * *$ &,$- 315^{* *}$ &,$- 291 * *$ &,$- 322 * *$ \\
\hline Distimia &,$- 652 * *$ &,$- 452 * *$ &,$- 507 * *$ &,$- 502 * *$ \\
\hline Disminución. Autoestima &,$- 627 * *$ &,$- 470 * *$ &,$- 553 * *$ &,$- 521 * *$ \\
\hline Flashbacks &,$- 325^{* *}$ &,$- 227 *$ &,- 185 &,$- 251 *$ \\
\hline Somatización &,$- 485^{* *}$ &,- 195 &,$- 232 *$ &,$- 234 *$ \\
\hline Autoimagen. negativa &,$- 606^{* *}$ &,$- 433^{* *}$ &,$- 497 * *$ &,$- 526 * *$ \\
\hline Autodesprecio &,$- 668 * *$ &,$- 422 * *$ &,$- 453 * *$ &,$- 509 * *$ \\
\hline
\end{tabular}

$* \mathrm{p} \leq .05$

$* * \mathrm{p} \leq .01$

En la tabla 2, se puede apreciar las correlaciones para el acoso escolar y la autoestima desde la relación negativa más alta es para autodesprecio y la autoestima general con coeficiente de -,688**, siendo este significativo; asimismo, se observa que en las otras dimensiones también existen una correlación significativa, lo que nos indica que existe una relación negativa entre las dimensiones de ambas variables. 


\section{DISCUSIÓN}

Respecto al aprendizaje autorregulado y la autoeficacia académica, se logró determinar una relación altamente significativa, esto confirma que en índices globales, el índice global acoso tiene una correlación alta con autoestima general $\left(-, 485^{* *}\right)$, Para ello se verificó con el aporte de Oñate (2007), en donde manifiesta que las consecuencias de la violencia escolar (la violencia escolar no es un buen escenario para el desarrollo de la autoestima) generan graves problemas psicológicos y sociales, son las víctimas ya que tienden a presentar conductas depresivas, baja autoestima, flagelación y aislamiento.

En escalas de acoso, manipulación social tiene una correlación alta con autoestima general $\left(-, 391^{* *}\right)$, cabe resaltar que si un adolescente mantiene un ambiente violento a nivel de pares, y el acoso es constante sus recursos personales se ven afectados, lo cual se van generando problemas psicológicos y emocionales, tales como dificultades para expresar lo que siente y lo que piensa, esquemas de pensamiento de desconfianza, abuso y asimismo imperfección, con la investigación de Tixe (2012), se puede apreciar que un $32 \%$ de estudiantes son víctimas de agresiones, determinando que la violencia escolar afecta la autoestima.

En Factores, el factor exclusión tiene una correlación alta con autoestima general $\left(-, 510^{* *}\right)$ y la escala social $\left(, 356^{* *}\right)$, el autoconcepto se va formando desde la primera infancia, los responsables de aquella formación son los padres, pero si a la vez el niño va creciendo en un ambiente donde se carece de fortalezas y sobresalen las debilidades su autoconcepto será bajo y disminuido, si adherido a ello un adolescente en su proceso educativo y su entorno estudiantil es criticado, burlado, excluido, intimidado, humillado y como consecuencia afecta sus recursos personales y autoconcepto.

La correlación entre las dimensiones de acoso escolar y autoestima en los estudiantes se puede apreciar que, en escalas clínicas, autodesprecio mantiene una correlación alta con la autoestima general $\left(-, 668^{* *}\right)$, Con la investigación de Sáenz, M. (2011) de tipo cuantitativa cuyo objetivo fue identificar la relación de sintomatología depresiva y acoso, encontró que sí existe relación entre la sintomatología depresiva y acoso escolar.

\section{CONCLUSIONES}

1. Las dimensiones de acoso escolar: índices globales, escalas de acoso, factores y escalas clínicas guardan una relación estadísticamente significativa con las dimensiones de autoestima.

2. A mayores situaciones de acoso escolar en el ambiente educativo menor es la autoestima de los adolescentes.

3. A mayores situaciones de índices globales de acoso escolar menor es la autoestima de los adolescentes.

4. A mayores situaciones de escalas de acoso escolar menor es la autoestima de los adolescentes.

5. A mayores situaciones de factores de acoso escolar menor es la autoestima de los adolescentes.

6. A mayores situaciones de escalas clínicas menor es la autoestima de los adolescentes.

7. Finalmente, no existe relación estadísticamente significativa entre la escala clínica de somatización y la autoestima social.

\section{REFERENCIAS}

Beltrán, J. \& Bueno, J. (1995). Psicología de la educación. Boixareu.

Cerezo, F. (2002). La violencia en las aulas: análisis y propuestas de intervención. Pirámide.

Cabeza, C. (2007). Violencia escolar: El acoso del profesor hacia el alumno. CreateSpace.

Cleary, M., Sullivan, G., \& Sullivan, K. (2003). Bullying: en la enseñanza secundaria. Ceac.

García, A. (2011). Violencia escolar y de género: conceptualización y retos educativos. Uhu.

Feldman,J. (2005). Autoestima: ¿cómo desarrollarla? juegos, actividades, recursos, experiencias creativas. Narcea. 
Gonzales-Arratia, N. (2001). La autoestima: medición $y$ estrategias de intervención a través de una experiencia en la reconstrucción del ser. UAEM.

González, V. (2009). Guía para el profesorado sobre acoso escolar: detección, identificación, intervención y prevención. Graficas Guiniguada.

Hernández, F. \& Sancho, M. (2004). El clima escolar en los centros de secundaria: más allá de los tópicos. Pardedós.

Izquierdo, C. (2008). Crecer en la autoestima. Paulinas.

Mendoza, M. (2012). Manual para Capacitar Profesionales en la Intervención y el Manejo de Víctimas de Acoso Escolar o Bullying. Copyright.

Olweus, D. (1998). Conductas de acoso y amenazas entre estudiantes. Morata.

Oñate, M. (2007). Mobbing escolar. Ceac.

OMS (2016). Estadísticas sanitarias mundiales 2014. OMS, 1(1), 45-50. http://www.who.int/gho/ indicator_registry/en/

Ortega, R., \& Del Rey, R. (2003). La Violencia Escolar: Estrategias de Prevención. Graó.

Osorio, F. (2006). Violencia en las escuelas: un análisis desde la subjetividad. Noveduc.
Porhola, M. \& Kinney, T. (2010). El acoso: Contextos, Consecuencias y Control. Aresta.

Ramos, M. (2008). Violencia y victimización en adolescentes escolares [Tesis de doctorado, Universidad Pablo de Olavide, Sevilla] https:// www.uv.es/lisis/manuel-ramos/tesis_ramos.pdf

Sáenz, M. (2011). Sintomatología depresiva y acoso escolar en un grupo de adolescentes escolares [tesis de licenciatura, Pontificia Universidad Católica del Perú] http://tesis.pucp.edu.pe/ repositorio/handle/123456789/677

Sánchez, H. \& Reyes, C. (2009). Metodología y Diseños en la Investigación Científica. Visión Universitaria.

Salm, R. (1999). La solución de conflictos en la escuela. Magisterio.

Tixe,D.(2013). La autoestima en adolescentes víctimas del fenómeno de Bullying [Tesis de licenciatura, Universidad Central del Ecuador] http://www. dspace.uce.edu.ec/bitstream/25000/3604/1/TUCE-0007-51.pdf

Vernieri, M. (2006). Adolescencia y Autoestima. Bonum.

Fecha de recepción: 24 de marzo 2021

Fecha de aceptación: 14 de julio 2021 